\title{
Teaching NeuroImages: Substantia nigra T2 hyperintensities in a man with Leber hereditary optic neuropathy
}

Karl Echiverri, MD, Kyle Darpel, MD, Charles Smith, MD, and Padmaja Sudhakar, MD

Neurology ${ }^{\circledR}$ 2019;93:e1830-e1831. doi:10.1212/WNL.0000000000008458

Figure MRI scans show symmetric substantia nigra T2 hyperintensities
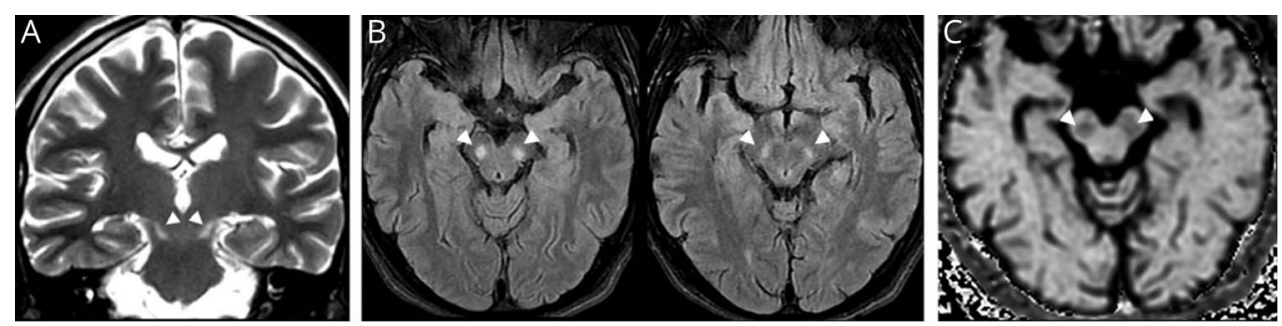

Coronal T2 MRI (A) and axial fluid-attenuated inversion recovery MRI (B) show bilateral symmetric substantia nigra T2 hyperintensities (arrowheads). These lesions do not show restricted diffusion (arrowheads) on axial diffusionweighted imaging (C).

A 29-year-old man with alcoholism presented with sudden progressive central visual loss in the right eye. Visual acuity was 20/300 (right) and 20/20 (left). Other findings included right relative afferent pupillary defect and bilateral hyperemic nonedematous optic discs concerning for OD retrobulbar optic neuritis. MRI head and orbit revealed symmetric T2 hyperintensities in the substantia nigra, without optic nerve hyperintensities (figure). Subsequently he lost vision in his left eye. Given that his maternal uncle developed bilateral optic neuritis in his 20s, genetic testing for Leber hereditary optic neuropathy was performed, finding a point mutation (m.11778G $>A$ ) in mitochondrially encoded NADH dehydrogenase 4 . The unusual gray matter hyperintensities in this case favored a metabolic rather than demyelinating etiology.

\section{Study funding}

No targeted funding reported.

\section{Disclosure}

The authors report no disclosures relevant to the manuscript. Go to Neurology.org/N for full disclosures.

\section{Correspondence}

Dr. Echiverri

Karl.echiverri@uky.edu

\section{MORE ONLINE}

$\rightarrow$ Teaching slides

links.lww.com/WNL/

A990 
Appendix Authors

\begin{tabular}{llll}
\hline Name & Location & Role & Contribution \\
\hline $\begin{array}{l}\text { Karl } \\
\text { Echiverri, } \\
\text { MD }\end{array}$ & $\begin{array}{l}\text { University } \\
\text { of } \\
\text { Kentucky }\end{array}$ & Author & $\begin{array}{l}\text { Drafting and revision, literature } \\
\text { review }\end{array}$ \\
\hline $\begin{array}{l}\text { Kyle } \\
\text { Darpel, }\end{array}$ & $\begin{array}{l}\text { University } \\
\text { of }\end{array}$ & Author & $\begin{array}{l}\text { Drafting and revision, literature } \\
\text { review }\end{array}$ \\
$\begin{array}{l}\text { KD } \\
\text { Charles }\end{array}$ & $\begin{array}{l}\text { University } \\
\text { of }\end{array}$ & Author & $\begin{array}{l}\text { Drafting and revision, literature } \\
\text { review, imaging acquisition and } \\
\text { formatting }\end{array}$ \\
\hline $\begin{array}{l}\text { Kadmaja } \\
\text { Sudhakar, }\end{array}$ & $\begin{array}{l}\text { University } \\
\text { of }\end{array}$ & Author & Drafting and revision \\
MD & Kentucky & & \\
\hline
\end{tabular}




\section{Neurology}

\section{Teaching NeuroImages: Substantia nigra T2 hyperintensities in a man with Leber hereditary optic neuropathy}

Karl Echiverri, Kyle Darpel, Charles Smith, et al.

Neurology 2019;93; $1830-\mathrm{e} 1831$

DOI 10.1212/WNL.0000000000008458

\section{This information is current as of November 4, 2019}

Updated Information \& Services

Subspecialty Collections

Permissions \& Licensing

Reprints including high resolution figures, can be found at: http://n.neurology.org/content/93/19/e1830.full

This article, along with others on similar topics, appears in the following collection(s):

Alcohol

http://n.neurology.org/cgi/collection/alcohol

Mitochondrial disorders

http://n.neurology.org/cgi/collection/mitochondrial_disorders

MRI

http://n.neurology.org/cgi/collection/mri

Optic nerve

http://n.neurology.org/cgi/collection/optic_nerve

Visual loss

http://n.neurology.org/cgi/collection/visual_loss

Information about reproducing this article in parts (figures,tables) or in its entirety can be found online at:

http://www.neurology.org/about/about_the_journal\#permissions

Information about ordering reprints can be found online:

http://n.neurology.org/subscribers/advertise

Neurology ${ }^{\circledR}$ is the official journal of the American Academy of Neurology. Published continuously since 1951, it is now a weekly with 48 issues per year. Copyright (O 2019 American Academy of Neurology. All rights reserved. Print ISSN: 0028-3878. Online ISSN: 1526-632X.

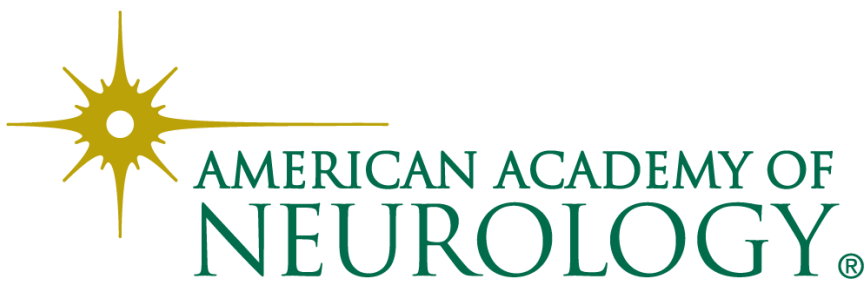

